\title{
Physiological Functions of Partially Hydrolyzed Guar Gum
}

\author{
Seon-Joo Yoon ${ }^{1, *}$, Djong-Chi Chu ${ }^{2}$, and Lekh Raj Juneja ${ }^{2}$ \\ ${ }^{1}$ Department of Pathobiology, Box 357238. HS Bldg, F 143, University of Washington, Seattle, WA 98195, USA \\ ${ }^{2}$ Central Research Laboratories, Taiyo Kagaku Co., Ltd., 1-3 Takaramachi, Yokkaichi, Mie 510-0844, Japan
}

Received 3 April, 2006; Accepted 12 July, 2006

\begin{abstract}
Summary Partially hydrolyzed guar gum (PHGG) has a number of properties associated with dietary fiber. PHGG ingestion results in not only an increase in defecting frequency and softer stools in persons with constipation but also significantly improvement of diarrhea in patient with gastrointestinal intolerance. The lowering of fecal pH by intake of PHGG resulted in the growth of Lactobacillus spp. and Bifidobacterium spp., intestinal flora good for human health. Improvement of balance of intestinal microflora resulted in prevention from infection and colonization of Salmonella enteritidis. Further the ingestion of PHGG promoted absorption of mineral and lowered serum cholesterol and triglycerides in the rat and serum cholesterol in human by improving lipid metabolism without reduction of protein utilization. In addition, PHGG significantly reduced the level of plasma glucose, and thereby improved acute postprandial plasma glucose and insulin response. All these observations suggest that the PHGG is prospective one of dietary fiber with various biological functions.
\end{abstract}

Key words: partially hydrolyzed guar gum (PGHH), intestinal microflora, mineral absorption, hyperlipidemia, glucose tolerance

\section{Introduction}

Dietary fibers, especially soluble fibers with high molecular weight, have several biological actions, including effect on slowing down of absorption of toxic substance in intestine, appetite suppression, attenuation of the plasma glucose and insulin response, lowering of plasma cholesterol level [1], slowing of the gut transit time with delaying digestion and macronutrients, bulking on the colon, anti-diarrhea nutrition support of colonocytes and colonic barrier function. They also could prevent the atrophy of small intestinal villi generated by long-term supplementation of liquid food [2].

Guar gum obtained from endosperm portion of Cyamoposis tetragonolobus is a galactomannan and considered as an excellent source for dietary fiber, because it can efficiently reduce the serum lipid and cholesterol level in healthy volunteers [3, 4], and hypercholesterolemic and hyperlipidemic subjects $[5,6]$. In addition, it has been very well known that guar gum improves glucose tolerance and prevents from diarrhea. Partially hydrolyzed guar gum (PHGG) produced by enzymatic hydrolysis using $\beta$-endo-mannanase has not only significant lower viscosity and molecular weight than intact guar gum but also similar biological action such as lowering of LDL cholesterol, lowering of blood glucose level, decrement of pancreatic enzyme activities, increment of fecal output, lowering of blood pressure, reducing of body weights, shortening of transit times in the gastrointestinal tract and inhibition of protein absorption. In this article, we reviewed the several biological functions of partially hydrolyzed guar gum (PHGG, product name: Sunfiber ${ }^{\circledR}$, produced by Taiyo Kagaku Co. Ltd., Japan).

\footnotetext{
*To whom correspondence should be addressed.

Tel: +1-206-860-6767 Fax: +1-206-726-1217

E-mail: yoonsj@u.washington.edu
} 
Effect of PHGG and Its Metabolites on Normalization of Physiological Condition Favor to Intestinal Microflora

Many papers have reported on the influence of guar gum on intestinal bacteria and enzymes in the human colon. It has been found that guar gum passes through digestion by mammalian intestinal enzymes ( $\alpha$-glucosidase, sucrase and maltase) but it is readily broken down to low molecular weight galactomannan in the large intestine by extracellular and membrane-bound glycolytic enzymes of the intestinal microflora and then used by intestinal bacteria as a carbon source $[7,8]$. It has also been clear that partial hydrolysis by bacterial endo- $\beta$-mannanase represents the first step in the metabolism of ingested guar gum. The reaction products are galactomannans of shorter chain length similar to those in PHGG. The in vitro studies on fermentation of PHGG and guar gum have been investigated in several studies $[9,10]$. These studies reported that several species of anaerobic bacteria isolated from human colon fermented some polysaccharides and consistently demonstrated that galactomannan was readily fermented by the fecal microflora [11].

The effects of PHGG on fecal microflora, bacterial metabolites and $\mathrm{pH}$ were investigated using nine healthy human volunteers administered 3 times a day with dose of $7 \mathrm{~g} /$ volunteer/day for 14 days [12]. The count of Bifidobacterium spp. and percentage of these species in the total count were significantly increased during the PHGG intake period. Among the acid-forming bacteria, Lactobacillus spp. also increased. It is well known that lactic-acid-forming bacteria have beneficial effects on human health by amino acid production, aiding defense activity against infection [13], pathogen inhibition [14], and immunopotentiation [15]. The $\mathrm{pH}$, content of bacterial metabolites (ex, ammonia) in fecal and $\beta$-glucuronidase activity were significantly decreased during PHGG intake period. These effects might be attributed to PHGG in human colon being consecutively degraded by certain bacteria for the degraded products to favor the growth of bifidobacteria and lactobacilli. The lowering of $\mathrm{pH}$ level induced the vigorous growth of bifidobacteria and lactobacilli in the intestine [16], resulted in improvement of intestinal condition by reducing formation of harmful bacterial metabolites [17], and reduced the ammonia content and $\beta$ glucuronidase activity $[18,19]$. Two weeks after the end of PHGG intake, the bacterial counts and their biological manifestations appeared to return to the former state. Another study suggested that PHGG prevented the formation of hard feces by, perhaps, inducing of adequate growth of several bacteria, and stimulated mucosa in small intestine by promoted the growth of mucosal cells with keeping its structure and enzyme activities [2]. All these observations suggest that the PHGG and/or its metabolites degraded by intestine microflora may contribute to normalization of physiological conditions favor to microflora in the colon, and thus can lead to moderation of constipation.

\section{Beneficial Effects of PHGG on Gastrointestinal Distress}

The consumption of some kinds of dietary fibers at recommended levels is generally not associated with any significant risk for human health. However, it has been well known that the ingestion of non-absorptive and fermentable carbohydrates with excessive amounts may cause laxation and gastrointestinal distress. Information on the tolerance of PHGG or guar gum is available from numerous clinical trials in which the beneficial effects on lipid and carbohydrate metabolism were investigated $[20,21]$. It has also been known that diarrhea induced by long-term consumption of a liquid diet was associated with the reduction in absorptive capacity and absorptive area due to the loss of brush border enzymes and mucosal cells [22]. The effects of two liquid diets with or without PHGG on intestinal function and microflora of rats were investigated [2]. Male Wistar rats, allocated into five groups: standard rats chow (MF), low-residue diets (LRD) and elemental diet (ED) with or without $1.5 \%$ PHGG at the dose of $60-70 \mathrm{kcal}$ daily for 2 weeks. The atrophy of terminal ileum villi was detected in LRD and ED groups. Supplementation with PHGG, however, improved the observed atrophy of terminal ileum $(p<0.05)$. The specific activity of diamine oxidase and alkaline phosphatase in mucosal scrapings was significantly lower in ileum of rats in LRD and ED groups than those in MF group. Those activities of rats supplemented with PHGG, however, significantly increased. Addition of PHGG to liquid diet suggested to improvement of the gastrointestinal tolerance and bowel control in long-term enterally fed patients.

\section{PHGG Demonstrates Preclusive Effect Against Diarrhea Through by Prolongation of the Colonic Transit Time and by Prevention of the Formation of Liquid in the Stools}

Gastrointestinal side effect, particularly diarrhea, is generally recognized as one of the most common complications associated with tube feeding after clinical practice in which diarrhea is attributed to infectious processes, antibiotic medication, protein energy malnutrition, patient-related causes such as stress and surgical procedures, and bacterial gut contamination [23]. It adds problems in patients care, disturbs fluid and electrolyte balance, and worsens the nutritional status. Therefore, diarrhea is considered as one of the main reasons for discontinuation of enteral nutrition. Its consequences are serious, and include infectious and skin care problems, loss of electrolytes, water and nutrients, and increase costs [24]. 
In human, dietary fiber is mainly degraded in the large intestine by bacterial flora, in which short-chain fatty acids (SCFAs) are liberated [25]. The SCFAs are absorbed by colon [26], which is associated with stimulation of sodium transport from the colon in several species including humans [27]. This effect may be particularly important in acute diarrheal diseases in colon and cause colonic dysfunction [28]. Luminal SCFAs levels in the colon may therefore influence the clinical course of acute diarrheal diseases. The fiber fermented to SCFAs by colonic bacteria was used for reducing diarrhea by adding with various forms to tubefeeding formulas, in which the action mechanism of fibers with regard to gastrointestinal effects seems highly dependent on the physical and chemical characteristics of the fibers themselves. It had reported that the children receiving either green plantain or pectin had significantly less stool output and duration of diarrhea [29]. However, soluble fiber such as guar gum has limiting factor to use in enteral formulas because its addition at physiologically effective concentrations results in the liquid products with high viscosity, thus its use in tube feeding is prevented. However, the advancement of technology on fiber and formula now made possible to addition of PHGG to enteral nutrition. It should be immediate concern to reveal whether PHGG would retain the beneficial physiological properties of guar gum and could safely be administered to patients in the form of clinical nutrition products. It was investigated whether surgical and medical patients enterally fed a formula diet enriched with PHGG showed much less diarrhea compared with patients given a standard formula without PHGG. Randomized and prospective double blind trial with 100 patients assessed the effects of feeding a standard diet vs the same diet supplemented with $20 \mathrm{~g} \mathrm{PHGG/l/day} \mathrm{[30].} \mathrm{The} \mathrm{results} \mathrm{indicated} \mathrm{that} \mathrm{the}$ occurrence of diarrhea under conditions of total enteral feeding or supplemental enteral feeding was significantly reduced when soluble $\mathrm{PHGG}$ was added to the enteral formula in a substantial amount $(2 \% \mathrm{wt} / \mathrm{v})$ (Fig. 1). It was also reported that PHGG prolonged colon transit time [31], by which the hydrolyzed form of guar gum offered the potential to reduce diarrhea and to positively influence the osmotic balance in the colon through increased fermentation by anaerobic bacteria that yield SCFA [32]. The effects of a standardized normal diet and two liquid formula diets with and without supplementation of PHGG $(21 \mathrm{~g} / l)$ on orocecal transit time, colonic transit time, bowel movements, and stool consistency were studied in 12 healthy male volunteers [31], in which the diets were consumed in a randomized order, each one for 7 days. The addition of PHGG did not affect orocecal transit time. Colonic transit time, however, was significantly prolonged $(55 \mathrm{~h})$ with PHGG supplementation compared with the liquid diet (39 h) and the self-selected diet $(30 \mathrm{~h})$. No effect of PHGG on large bowel function was seen and no complaints about

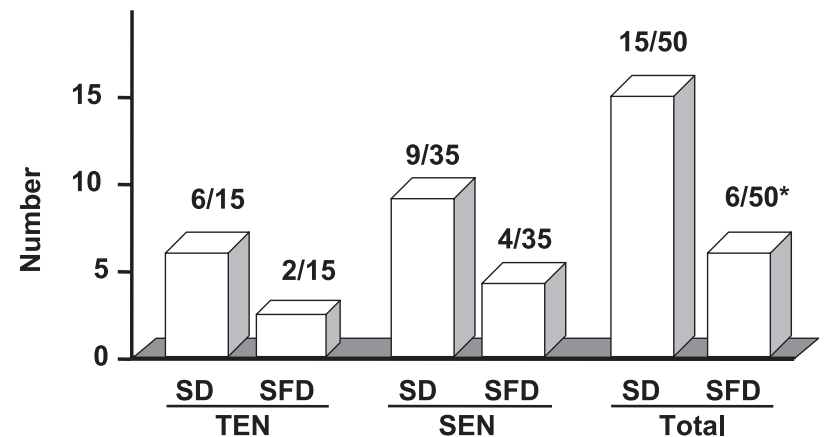

Fig. 1. Effect of PHGG on diarrhea incidence [30]. Twenty grams a day of PHGG was administered to patient with gastrointestinal intolerance. SD, Standard liquid; SFD, PHGG diet; TEN, Total enteral nutrition; SEN, Supplemental enteral nutrition; Total, TEN + SEN . Each datum shows diarrhea/total subjects. Statistical analysis of the data was carried out using the Statview program. For comparison of diarrhea incidence during nutrition, $X^{2}$ test and Fisher's Exact Test were used; significance was assumed if $* \mathrm{p}<0.05 . \mathrm{n}=50$.

gastrointestinal side effects were received. Though stool frequency and consistency were not different between the groups with and without PHGG, neither diarrhea nor constipation was observed in the group with PHGG. PHGG was also well tolerated when given to eight healthy men with beverage formula after each meal for a period of 4 weeks [33]. None of the healthy volunteers complained diarrhea or unacceptable gastrointestinal symptoms. Alam et al. [34] reported that PHGG added to standard WHO ORS (World Health Organization Oral Rehydration Solution) substantially reduced the duration of diarrhea and modestly reduced stool output in acute noncholera diarrhea in young children, and Spapen et al. [35] suggested that total enteral nutrition supplemented with PHGG was beneficial in reducing the incidence of diarrhea in tube-fed full-resuscitated and mechanically ventilated septic patients. Although the underlying mechanism of action of PHGG in reducing the incidence of diarrhea in enteral nutrition is not entirely clear, two effects are considered as of major importance. First, the soluble fiber significantly prolongs the colonic transit time, which is apparently correlated with basally increased cholecystokinin concentration. Second, PHGG seems to be fermented by colonic bacteria to SCFAs in a way that increases the luminal concentration and absorption of SCFA in the colon, which promotes the absorption of sodium and water by the colonocytes [36]; and, in turn, leads to a decreased fluidity in the lumen and may prevent the formation of liquid in the stools [37]. In conclusion, PHGG is potential candidate for a new antidiarrheal therapy for acute diarrhea. 


\section{PHGG Improves the Constipation Condition with Increasing the Defecation Frequency}

Constipation defined as a symptom of hard feces causes difficulty of output due to delayed fecal transit time [38]. Constipation occurs when there is no fecal output for 3 or 4 days and followed by a pain. It is also one of the first symptoms to be classified as a fiber deficiency disorder and frequently found in chronic care patients who are fed exclusively on enteral formula. Dietary fiber has been considered to be an effective to regulate fecal transit time by reducing both constipation and diarrhea, therefore it improves defecation by fecal bulking, consistency and increasing of intestinal motility [39]. It has been reported that intake of $20 \mathrm{~g}$ of guar gum a day increases the fecal weight of healthy volunteers by $20 \%$ [25]. However, intake of $11.4 \mathrm{~g}$ guar gum a day resulted in no increase in the fecal output [4]. The effect of fiber intake on fecal bulk was reported to relate not only with the water-holding capacity of the surviving residue, but also to stimulation of bacterial growth [40]. As regards to effect of PHGG on fecal bulking, it was found that the fecal output was increased up to $42 \%$ when $36 \mathrm{~g}$ of PHGG per day was administered to healthy volunteers [33], and significantly increased in rats fed diets with 2.5 and 5\% PHGG. The influence of PHGG on constipation was investigated on women who commonly felt considerable discomfort before movements, suffered abdominal pain and their fecal output occurred on an average of 2.8 times per week [41]. In the experiment, $11 \mathrm{~g}$ of PHGG per day was administered as a beverage formula to fifteen constipated women for 2 and 3 weeks, and then defecating frequency, $\mathrm{pH}$, weight, moisture and bacterial flora of the feces were investigated during the entire experiment periods. The defecating frequency during one day increased from $0.46 \pm 0.05$ to $0.66 \pm 0.05$ (Fig. 2) as well as fecal moisture was significantly increased from $69.1 \%$ to $73.8 \%$. Fecal moisture content was increased consistently with lowering the $\mathrm{pH}$ of feces $(\mathrm{r}=-0.478)$. The frequency of Lactobacillus spp. occurrence in feces was also significantly increased by the ingestion of PHGG compared with the control period. These results indicate that SCFAs might be produced by fermentation of PHGG and lowered the fecal $\mathrm{pH}$ [42], and then low $\mathrm{pH}$ in the large intestine leaded to the stimulation on growth of Lactobacillus spp. [43]. Therefore, the observed increase in fecal moisture at a low $\mathrm{pH}$ may be due to the growth of Lactobacillus spp. In another study, fecal output was also increased in human volunteers ingesting PHGG both at a dose of $36 \mathrm{~g} /$ day for 4 weeks [44] and $12 \mathrm{~g} / 100 \mathrm{ml}$ beverage/day for 12 weeks [33]. A clinical study has also showed that PHGG was effective in preventing constipation by increasing bulking capacity in the institutionalized elderly women and men who took laxatives on a regular basis. The results of those studies

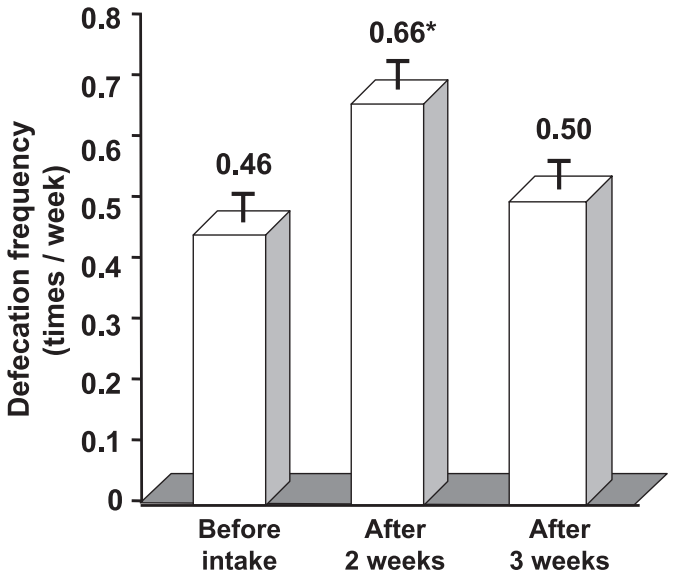

Fig. 2. Effect of PHGG on defecation of each week in constipated women [41]. PHGG was administered as beverage (11 g a day) to 15 females suffering from constipation for 3 weeks, and then defecation frequency per a day was investigated. Each datum represents average of values from all women. The significance between mean was determined by the student $t$-test $\left({ }^{*} \mathrm{p}<0.05\right.$.)

concluded that PHGG should be considered in place of laxatives for elderly women and men who found traditional fiber supplements unpalatable, or for people who needed fiber added to their tube-feeding formula [45]. As a result, it can be concluded that administration of PHGG contributes to soft feces, thus increasing the defecation frequency, and restoring the balance of intestinal microflora, and results in improvement of diarrhea and constipation condition.

\section{PHGG and/or Its Metabolites Improve the Efficacy of Iron Utilization in Patients Suffering from Iron Deficiency}

The maintenance of iron homeostasis and prevention of iron deficiency anemia require controlled absorption of iron to balance uncontrolled body losses. Iron deficiency anemia occurs as a result of blood loss, urinary iron loss with intravascular hemolysis, pregnancy, inadequate dietary intake or malabsorption of iron [46]. It has been known that sorbitol [47], bile [48] and those substances increase iron absorption in the small intestine in man [49]. It was reported that guar gum interfered the absorption of $\mathrm{Zn}, \mathrm{Cr}, \mathrm{Cu}$ and $\mathrm{Co}$ but not of $\mathrm{Fe}$ and $\mathrm{Ca}$, in a rat experiment [50], of which mechanism is still unknown but may be explained by a high adhesive effect of high viscous guar gum [51].

When the effect of PHGG on iron absorption and utilization was investigated in growing rats fed several iron-deficient diets, it was demonstrated that PHGG and its metabolites increased the bioavailability of ferrous iron [52]. The ferrous iron concentrations of hemoglobin and serum in rats of the groups fed diets supplemented with PHGG were at similar 
levels to those of the rats fed an iron-sufficient diet. The storage of iron in liver and spleen of the rats fed PHGG was greater than that of the rats grown on an iron-deficient control diet. Those facts indicate that the change in the level of iron component of blood is not due to iron stored in spleen or liver but due to change in ability of iron utilization in the diet. Administration of PHGG also caused the increment of iron adsorption on intestinal villi in an iron balance experiment for 3 days, which was likely that the addition of PHGG into liquid diets had reduced shrinkage of ileum villi in rat intestine [53]. As result, $\mathrm{PHGG}$ and/or its metabolites might be effective to improve the efficacy of utilization of ferrous iron in the subjects suffering from iron deficiency as a result from blood loss with menses, urinary iron loss with intravascular hemolysis, pregnancy, inadequate dietary intake or malabsorption of iron. In another study, it was reported that PHGG promoted absorption of calcium and magnesium in rat [54] (Fig. 3). The result also showed that calcium absorption in five-sixths nephrectomized (NPX) rats was considerably lower than that in normal rats in the PHGG-free groups, but the absorption in PHGG-fed NPX rats was slightly lower than that in normal PHGG-fed rats, and that the caecum was responsible for these increases in calcium absorption and a considerable amount of calcium was absorbed from the large intestine in PHGG-fed rats. They, therefore, suggested that nephrectomy does not influence the absorption of calcium in the large intestine induced by PHGG feeding and the increase in the caeco-colonic absorption compensates the decreasing proximal intestinal calcium transport associated with nephrectomy. The increment of mineral absorption associated with PHGG feeding may be attributed to gut acidification resulting from caecal fermentation of dietary fiber, which consequently elevated the level of ionic calcium in the large intestine.

\section{PHGG is Effective in Decreasing Body Fat and Energy Deposition without Reduction of Protein Uti- lization}

Dietary fibers such as pectin and guar gum are known to affect the digestibility and absorption of several nutrients including protein and lipid. Amount of dietary fiber indigested has been demonstrated increment of secretion of faecal nitrogen as well as decrement of digestibility of dietary protein and protein utilization in both animal and human [55]. It has been reported that guar gum decreases the utilization efficacy of protein [56] and lipid [6], which may be explained by the high viscosity of guar gum dissolved in water which results in a slower gastric emptying [57], interfering with the digestion of food and absorption of nutrients. In study fed the diets containing intact guar gum and varying amount of PHGG (5 and 10\%) to growing rats for 3 weeks, food consumption was almost similar to that of
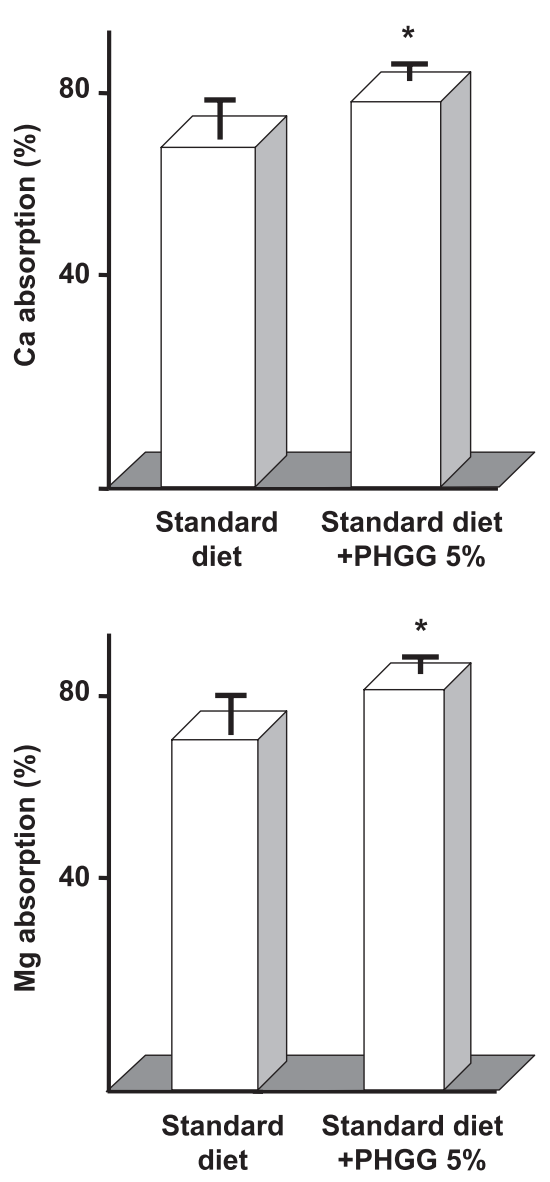

Fig. 3. Enhancement of mineral absorption by PHGG [54]. The PHGG (50 g/kg diet) was added to a test diet containing $3.0 \mathrm{~g}$ mineral $/ \mathrm{kg}$ diet at the expense of whole diet and then administered to 6 rats. A, Ca absorption and $\mathrm{B}, \mathrm{Mg}$ absorption. The mineral absorption (\%) was calculated as follows; $\mathrm{Ca}(\mathrm{Mg})$ absorption $(\%)=100 \times($ total $\mathrm{Ca}$ $(\mathrm{Mg})$ intake - faecal $\mathrm{Ca}(\mathrm{Mg})$ excretion)/total $\mathrm{Ca}(\mathrm{Mg})$ intake. Each datum line represents average \pm SEM of values of 6 rats $(* \mathrm{p} 0.05)$.

the control rats except that the rats fed guar gum gradually declined [58]. The value for digestible and metabolizable energy and efficiency of energy utilization declined in the rats fed the experimental diets but protein utilization remained unchanged. The results suggest that PHGG is effective in decreasing body fat and energy deposition without reduction of protein utilization.

\section{PHGG Leads to Lowering Effect of Cholesterol and Triglyceride Level in Serum by Stimulating the Production of Short-chain Fatty Acids}

Improvement of lipid metabolism such as cholesterol and triglyceride believed as a key risk factor to the prevention and treatment of heart disease and arteriosclerosis has been 
thought effective in preventing and treating some kinds of adult metabolic diseases. For this purpose, dietary fiber had drawn considerable attention to improve lipid metabolism [59]. It has been well demonstrated that various kinds of water-soluble dietary fiber improve lipid and carbohydrate metabolism [60, 61], and show potent hypocholesterolemic effects in experimental animals and in humans [39]. There were reports that guar gum diets significantly reduced serum cholesterol (low-density lipoprotein, LDL) level in normal and hyperlipidemic subjects [4], while maintained highdensity lipoprotein (HDL) cholesterol level [62]. Also, guar gum and some other gel forming dietary fibers have been demonstrated to lower the hypolipidemic and blood cholesterol level in a feeding study with rats and in a study with healthy volunteers $[63,64]$. There was a consensus that the high polymeric structure was a prerequisite for these non-absorbable carbohydrates to exert a potent cholesterollowering effect [65]. However, it has been reported that fructooligosaccharide, a mixture of nonabsorbable oligosaccharides consisting of less than 5 sugar units, could reduce the serum cholesterol in diabetic subjects [66]. PHGG was also effective in reducing serum triacylglycerol as well as cholesterol levels in the rats fed high-fat diets employing lard or palm oil as diet fat [1], in which total lipids and cholesterol were increased in the rat feces but decreased in the rat liver. Yamatoya et al. [67] reported that intake of PHGG significantly reduced the concentration of serum cholesterol and free fatty acid as well as serum triglyceride and phospholipid. In human volunteer study, total serum cholesterol was reduced by the daily administration of 3 serves of each $12 \mathrm{~g}$ of PHGG a day while other serum lipid parameters remained unaffected [33] (Fig. 4). These studies led a suggestion that levels of cholesterol and triglyceride in both serum and liver were dose-dependently decreased, thereby PHGG might be applied for a hypocholesterolemic agent. The effectiveness on cholesterolemia might be explained that PHGG entraps the bile acids in the small intestine by interruption of the entero-hepatic circulation of bile salts and increases their fecal excretion, which accelerates cholesterol oxidation to bile acids, resulting in a spillover of the body cholesterol pool, due to fecal losses of steroids.

Bacterial fermentation of dietary fiber in the large intestine leads to the production of SCFAs mainly acetic acid, propionic acid and butyric acid. Some gases such as hydrogen, carbon dioxide and methane are also produced to a lesser extent [68]. It has been demonstrated that the SCFAs, propionic acid and butyric acid were significantly produced by colonic microflora in the presence of oligosaccharide and hydrolyzed guar gum [69], and that the plasma cholesterol lowering effects of soluble fiber might be related to the propionic acid and other microbial metabolites produced in the gut [70]. The SCFAs are known to be rapidly absorbed by the colonic mucosa stimulating the mucosal blood flow,

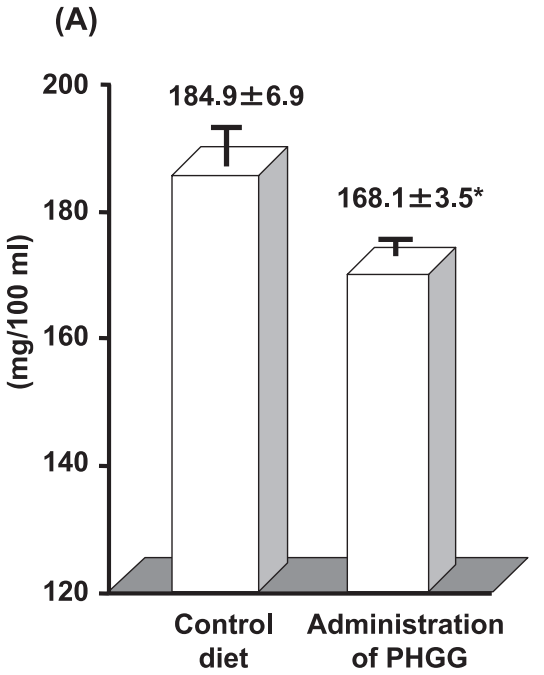

(B)

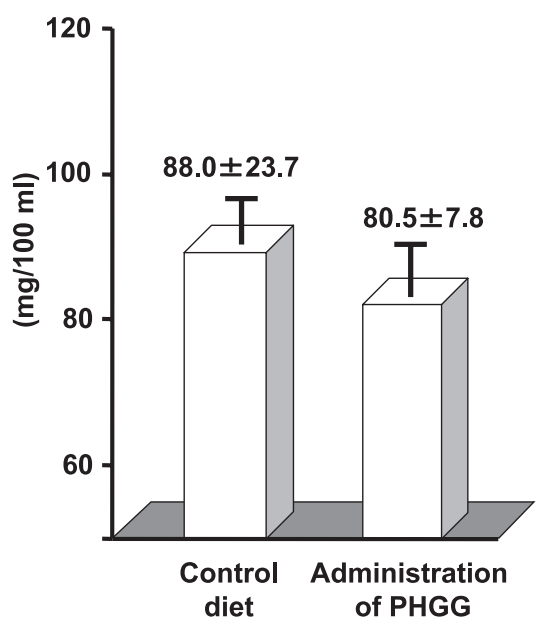

Fig. 4. Hypolipidemic effects of PHGG [33]. The cholesterol (A) and triglyceride (B) levels in serum were determined in 8 healthy males given 3 times a days with the control diet plus the PHGG (12 g PHGG/100 ml) for 4 weeks post prandial. Each datum represents average of values \pm SEM of 8 persons. The significance between mean values was determined by the Duncan's multiple range test $\left({ }^{*} \mathrm{p}<0.05\right)$.

epithelial proliferation of large intestine, mucus secretion and absorption of water, $\mathrm{Na}, \mathrm{Ca}$ and $\mathrm{Mg}$ from the large intestine as well as peristalsis, thus aiding with homeostasis and bowel function [71]. Rat experiment fed PHGG with dose of $5 \%$ and $10 \%$ showed the 2-3 times higher content of FCFAs compared with that of control rats in cecum, which study also demonstrated that PHGG significantly produced the highest levels of total SCFAs in comparison with other materials such as glucose, fructooligosaccharide, inulin, hydrolyzed inulin and cullulose [69] (Fig. 5). From these results, PHGG might improve lipid metabolism and 


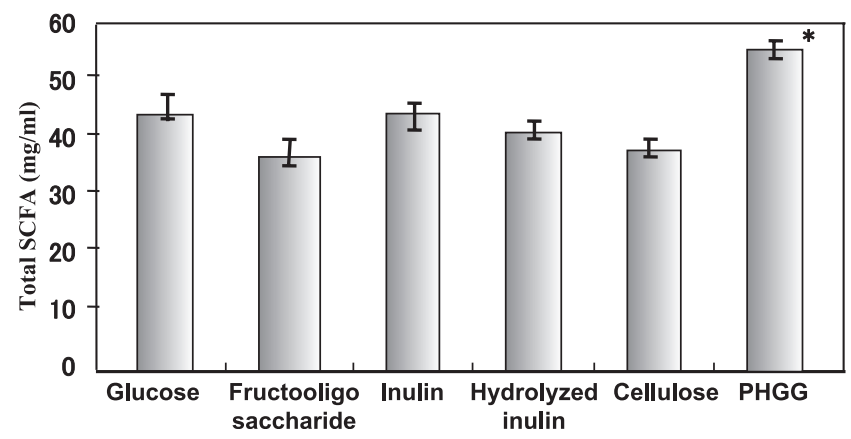

Fig. 5. Production of total short-chain fatty acids (SCFAs) as the main by-products of dietary fiber and oligosaccharide microbial fermentation [69]. The $0.5 \mathrm{~g}$ of each material was hydrated and then inoculated with the faecal homogenate. After fermentation for $24 \mathrm{~h}$ at $37^{\circ} \mathrm{C}$ in water bath, SCFAs concentration of aliquots was measured. The effects of different commercial oligosaccharides and fiber substitutes on SCFA production as compared with the control glucose and among substrates were determined using analysis variance (NOVA) followed by the Scheffe F-test of significance using StatView 1.02 software (SE + Graphics, Abacus Concepts, Inc.). A p value of $<0.05$ was considered to indicate a significant difference between the test fibers and the control. Each datum line represents average of values \pm SEM of 8 persons.

lead to lower the cholesterol and triglyceride levels in serum.

\section{PHGG Improve the Acute Post-prandial Plasma Glucose and Insulin Response in the Study on Glucose Tolerance in Non-insulin-dependent Diabetic Patients}

Hospitalized patients often require nutritional supplementation for maintenance of adequate nutrition [72], which, however, often causes hyperglycemia in glucose intolerant and diabetic patients. Therefore, it is considered as most difficult point in blood glucose management. Soluble dietary fibers such as guar gum and pectin have been demonstrated effective in the treatment of post-prandial glycemia in both normal and diabetic subjects, often coupled with a reduced need for insulin secretion [73]. Considering these physiological benefits, it appears logical to enrich liquid enteral formulas with soluble fiber to create products suitable for diabetic and glucose intolerant patients requiring supplementation. However, addition of soluble fiber to liquid products with physiologically effective concentrations causes a substantial increase viscosity and prevents easy oral consumption or application by gastrostomy tube. Thus, the use of PHGG makes possible to decrease the viscosity in comparison with intact guar gum and allows the production of liquid products suitable for oral supplementation or application via gastrostomay tube. The effect of liquid supplement containing PHGG on post-prandial glucose and insulin excursions in 6 non-insulin-dependent diabetic (NIDDM) patients was reported, of which result demonstrated that the enteral formula diet containing PHGG resulted in significant improvements of acute post-prandial glucose and insulin response [74] (Fig. 6). When glucose tolerance in NIDDM was examined both in rats and human volunteers, enteral liquid meal containing PHGG, demonstrated significant improvements in lowering plasma glucose and insulin response [75-77], of which effect was not likely exclusively mediated through viscosity increases resulting in delayed gastric emptying [74]. In another study, effects of PHGG on elevation of blood glucose and on insulin secretion after sucrose intake were investigated using healthy human volunteers. When $30 \mathrm{~g}$ sucrose was taken, the peak of blood glucose and insulin levels was recognized at $30 \mathrm{~min}$ after ingestion. When $5 \mathrm{~g}$ of PHGG was taken simultaneously with $30 \mathrm{~g}$ sucrose, blood glucose level at $60 \mathrm{~min}$ after ingestion was significantly lower $(p<0.05)$ than that of sucrose alone. Serum insulin level was also lower than that of the control [77]. These data show that viscosity of PHGG is greatly lower than that of intact guar gum, but the reduction effect of blood glucose level after sucrose intake was remained. Therefore, acute glucose lowering effect of guar gum is not solely explained by delayed gastric emptying in the delivery of viscous material from the stomach into the small bowel $[61,78]$. PHGG showed the significant improvement of acute post-prandial plasma glucose and insulin response in the study on glucose tolerance in non-insulin-dependent diabetic patients.

\section{PHGG Contributes to Improvement of Balance of Intestinal Microflora by Preventing from the Infection and Colonization of Salmonella enteritidis (SE)}

Serious contamination by Salmonella spp. both in and on eggs has been reported in Europe, US [79] and Japan [80]. The infection of SE in poultry is one of the most serious problems in the poultry industry because of related outbreaks of food poisoning [81, 82]. It has been reported that some kinds of carbohydrates, yeast and especially the mannose residue are effective in preventing the Salmonella 
(A)

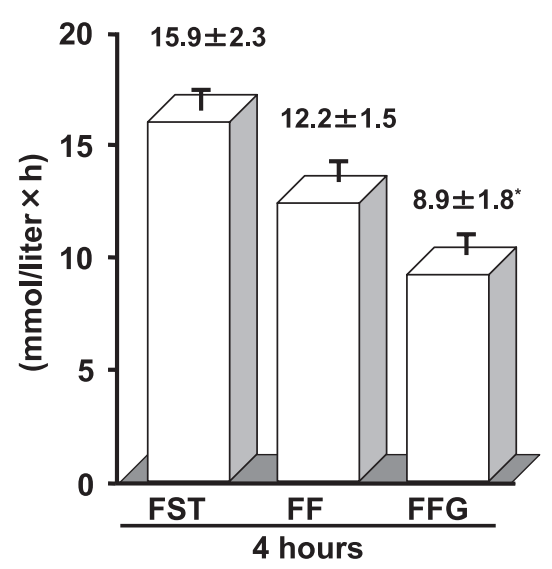

(B)

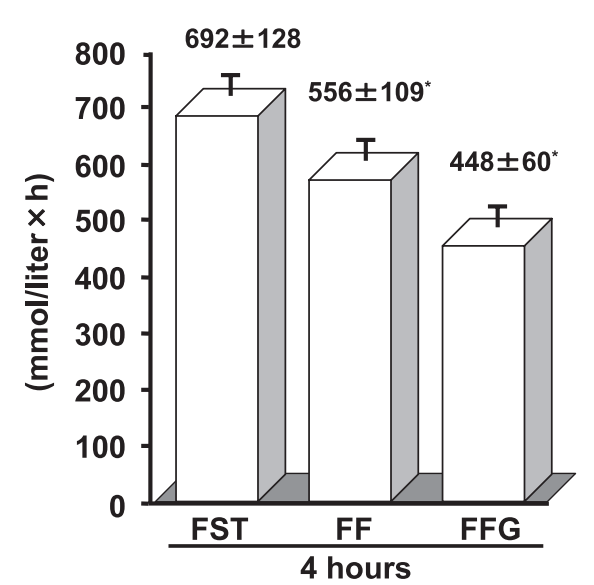

Fig. 6. Effect of PHGG on glucose tolerance in non-insulindependent diabetic patients [74]. The levels of glucose (A) and insulin (B) in plasma were investigated in 6 noninsulin-dependent diabetic patients. The levels of glucose and insulin in plasma were determined after $4 \mathrm{~h}$ following consumption of $430 \mathrm{ml}$ of FST, or $500 \mathrm{ml}$ of FF or FFG. FST, sucrose containing standard enteral formula diet (a fiber-free); FF, fructose containing enteral formula diet (a fiber-free); FFG, FF with the addition of $20 \mathrm{~g}$ PHGG per liter. Each datum represents average of values \pm SEM of 6 persons. The significance between mean values was determined by the Wilcoxon signedrank test $(* \mathrm{p}<0.05)$.

spp. colonization [83, 84]. The preventive effect of PHGG on the colonization of SE was investigated in SE-infected young and laying hens fed orally the supplement containing 0.025 , 0.05 , and $0.1 \%$ PHGG. The incidence of SE in organs and agglutinating antibody titer to SE in serum were decreased by the administration of PHGG to young hens, while the excretion of SE to feces was increased. The experiment demonstrated significant increment of Bifidobacterium spp. and Lactobacillus spp., the most numerous useful intestinal bacteria in the cecum of young hen, and supplementation with $0.025 \%$ PHGG was the most effective. The effect of the excretion of SE via feces was also observed in an experiment using laying hens. The incidence of SE in the surface of the egg shell, egg white and egg yolk was decreased when the feed of laying hens was supplemented with $0.025 \%$ PHGG. These results suggest that the administration of feed supplement with PHGG can prevent the colonization of SE in young and laying hens, which, in turn, might be related to improvement in the balance of intestinal microflora, as observed in rat and human.

\section{References}

[1] Ide, T., Moriuchi, H., and Nishimoto, K.: Hypolipidemic effects of guar gum and its enzyme hydrolysate in rats fed highly saturated fat diets. Ann. Nutr. Metab., 5, 34-44, 1991.

[2] Takahashi, H., Akachi, S., Ueda, Y., Kim, M., Hirano, K., and Yamamoto, T.: Effect of liquid diets with or without partially hydrolyzed guar gum on intestinal microbial flora and function of rats. Nutr. Res., 15, 527-536, 1995.

[3] Jenkins D.J.A., Leeds, A.R., Newton, C., and Cummings, J.H.: Effect of pectin, guar gum and wheat fiber on serum cholesterol. Lancet, 1, 1116-1117, 1975.

[4] Penagini, R., Velio, P., Vigorelli, R., Bozzani, A., Castagnone, D., Ranzi, T., and Bianchi, P.A.: The effect of dietary guar on serum cholesterol, intestinal transit and fecal out put in man. Am. J. Gastroenterol., 81, 123-125, 1986.

[5] Jenkins, D.J.A., Leeds, A.R., Slavin, B., Mann, J., and Jepson, E.M.: Dietary fiber and blood lipids: reduction of serum cholesterol in type II hyperlipidemia by guar gum. Am. J. Clin. Nutr., 32, 16-18, 1979.

[6] Simons, L.A., Gayst, S., Balasubramaniam, S., and Ruys, J.: Long-term treatment of hypercholesterolaemia with a new palatable formulation of guar gum. Atherosclerosis, 45, 101108, 1982.

[7] Tomlin, J., Read, N.W., Edwards, C.A., and Duerden, B.I.: The degradation of guar gum by a fecal incubation system. Br. J. Nutr., 55, 481-486, 1986.

[8] Balascio, J.R., Pallmer, J.K., and Salyers, A.A.: Degradation of guar gum by enzymes produced by a bacterium from the human colon. J. Food Biochem., 5, 271-282, 1981.

[9] Gibson, G.T., Macfarlane, S., and Cummings, J.H.: The fermentability of polysaccharides by mixed human fecal bacteria in relation to their suitability as bulk-forming laxatives. Let. Appl. Microbiol., 11, 251-254, 1990.

[10] McBurney, M.I. and Thompson, L.U.: In vitro fermentabilities of purified fiber supplements. J. Food Sci., 54, 347-350, 1989.

[11] Salyers, A.A., West, S.E.H., Vercellotti, J.R., and Wilkins, T.D.: Fermentation of mucins and plant polysaccharides by anaerobic bacteria from the human colon. Appl. Environ. Microbiol., 34, 529-533, 1977.

[12] Okubo, T., Ishihara, N., Takahashi, H., Fujisawa, T., Kim, M., Yamamoto, T., and Mitsuoka, T.: Effects of partially hydrolyzed guar gum intake on human intestinal microflora 
and its metabolism. Biosci. Biotech. Biochem., 58, 13641369, 1994.

[13] Hentges, D.J.: Role of the intestinal microflora in host defense against infection, in Human intestinal Microflora in Health and Disease, ed. By Hentges, D.J., Academic Press, New York, pp. 311-331, 1983.

[14] Bullen, J.J., Rogers, H.J., and Leigh, L.: Iron-binding proteins in milk and resistance to Escherichia coli infection in infants. Br. Med. J., 1, 69-75, 1972.

[15] Berg, R.D.: Host immune response to antigens of the indigenous intestinal flora, in Human Intestinal Microflora in Health and Disease, ed. By Hentges, D.J., Academic Press, New York, pp. 101-126, 1983.

[16] Willis, A.T., Bullen, C.L., Williams, K., Fagg, C.G., Bourne, A., and Vignon, M.: Breast milk substitute: a bacteriological study. Br. Med. J., 4, 67-72, 1973.

[17] Pietroiusti, A., Caprilli, R., Giuliano, M., Serrano, S., and Vita, S.: Fecal pH in colorectal cancer. Ital, J. Gastroenterol., 17, 88-91, 1985.

[18] Mallet, A.K., Rowland, I.R., Bearne, C.A, Flynn, J.C., Fehilly, B.J., Udeen, S., and Farthing, M.J.G.: Effect of dietary supplements of apple pectin, wheat bran or fat on the enzyme activity of the human faecal flora. Microbial Ecol. Health Disease, 1, 23-29, 1988.

[19] Sugawara, M., Suzuki, K., Endo, K., Kumemura, M., Takeuchi, M., and Mitsuoka, T.: Effect of dietary supplementation of corn hemicellulose on fecal flora and bacterial enzyme activities in human adults. Agric. Biol. Chem., 54, 16831688, 1990.

[20] Todd, P.A., Benfied, P., and Goa, K.L.: Guar gum. A review of its pharmacological properties, and use as a dietary adjunct in hypercholesterolaemia. Drugs, 39, 917-928, 1990.

[21] McIvor, M.E., Cummings, C.C., and Mendeloff, A.I.: Longterm ingestion of guar gum is not toxic in patients with non-insulin-dependent diabetes mellitus. Am. J. Clin. Nutr., 41, 891-894, 1985.

[22] Hosoda, N., Nishi, M., Nakagawa, M., Hiramatsu, Y., Hioki, K., and Yamamoto, M.: Structural and functional alterations in the gut of parenterally or enterally fed rats. J. Surg. Res., 47, 129-133, 1989.

[23] Bliss, D.Z., Guenter, P.A., and Settle, R.G.: Defining and reporting diarrhea in tube-fed patients; What a mess. Am. J. Clin. Nutr., 55, 753-759, 1992.

[24] O'Brien, B.L.: Diarrhea in the critically ill. Cur. Option Critical Care, 2, 140-144, 1996.

[25] Cummings, J.H., Branch, W., and Jenkins, D.J.A.: Colonic response to dietary fiber from carrot, cabbage, apple, bran and guar gum. Lancet, 1, 5-9, 1978.

[26] Ruppin, H., Bar-Meir, S., Soergel, K.H., Woo, C.H., and Schmitt, M.G. Jr.: Absorption of short-chain fatty acids by the colon. Gastroenterology, 78, 1500-1507, 1980.

[27] Roediger, W.E. and Moore, A.: Effect of short-chain fatty acid on sodium absorption in insolated human colon perfused through the vascular bed. Dig. Dis. Sci., 26, 100-106, 1981.

[28] Ramakrishna, B.S. and Mathan, V.I.: Colonic dysfunction in acute diarrhea: the role of luminal short chain fatty acids. Gut, 34, 1215-1218, 1993.
[29] Rabbani, G.H., Fuchs, G.J., and Teka, T.: Beneficial effects of pectin and raw banana in the dietary management of persistent diarrhea in children. Gastroenterology, 114, A407, 1998.

[30] Homann, H.H., Kemen, M., Fuessenich, C., Senkal, M., and Zumtobel, V.: Reduction in diarrhea incidence by soluble fiber in patients receiving total or supplemental enteral nutrition. J. Parenteral Enteral Nutr., 18, 486-490, 1994.

[31] Meier, R., Beglinger, C., Schneider, H., Rowedder, A., and Gyr, K.: Effect of liquid diet with and without soluble fiber supplementation on intestinal transit and cholecystokinin release in volunteers. J. Parenteral Enteral Nutr., 17, 231235, 1993.

[32] Palacio, J.C. and Rombeau, J.L.: Dietary fiber: A brief review and potential application to enteral nutrition. Nutr. Clin. Pract., 5, 99-106, 1990.

[33] Takahashi, H., Yang, S.I., Hayashi, C., Kim, M., Yamanaka, J., and Yamamoto, T.: Effect of partially hydrolyzed guar gum on fecal output in human volunteers. Nutr. Res., 13, 649-657, 1993.

[34] Alam, N.H., Meier, R., Schneider, H., Sarker, S.A., Bardhan, P.K., Mahalanabis, D., Fuchs, G.J., and Gyr, N.: Partially hydrolyzed guar gum-supplemented oral rehydration solution in the treatment of acute diarrhea in children. J. Pediatr. Gastroenterol. Nutr., 31, 503-507, 2000.

[35] Spapen, H., Diltoer, M., Van Malderen, C., Opdenacker, G., Suys, E., and Huyghens, L.: Soluble fiber reduces the incidence of diarrhea in septic patients receiving total enteral nutrition: a prospective, double-blind, randomized, and controlled trial. Clin. Nutr., 20, 301-305, 2001.

[36] Roediger, W.E. and Rae, D.: Trophic effect of short-chain fatty acids in mucosal handling of ions by the defunctioned colon. Br. J. Surg., 69, 23-25, 1982.

[37] Zimmaro, D.M., Rolandelli, R.H., Koruda, M.J., Settle, R.G., Stein, T.P., and Rombeau, J.L.: Isotonic tube-feeding formula induces liquid stool in normal subject: Reversal by pectin. $J$. Parenteral Enteral Nutr., 13, 117-123, 1989.

[38] Sakata, T.: Function of uppergastrointestine: Bases in digestion and absorption, in Digestion and Absorption, ed. By Muto, Y., Dai-ichi Shuppan Publishing Co. Ltd., Tokyo, pp. 145161, 2002.

[39] Kay. R.M.: Dietary fiber. J. Lipid Res., 23, 221-242, 1982.

[40] Stephen, A.M. and Cummings, J.H.: Mechanism of action of dietary fibre in the human colon. Nature, 284, 283-284, 1980.

[41] Takahashi, H., Wako, N., Okubo, T., Ishihara, N., Yamanaka, J., and Yamamoto, T.: Influence of partially hydrolyzed guar gum on constipation on women. J. Nutr. Sci. Vitaminol., 40, 251-159, 1994.

[42] Mitsuoka, T., Ohno, K., Benno, Y., Suzuki, K., and Namba, K.: The fecal flora of man IV. Communication: Comparison of the newly developed method with the old conventional method for the analysis of intestinal flora. Hyg. I. Abt. Orig., A234, 219-233, 1976.

[43] Hood, S.K. and Zottola, E.A.: Effect of low $\mathrm{pH}$ on the ability of Lactobacillus acidophilus to survive and adhere to human intestinal cells. J. Food Sci., 53, 1514-1516, 1988. 
[44] Shankardass, K., Chuchmach, S., Chelswick, K., Stefanovich, C., Spurr, S., Brooks, J., Tsai, M., Saibil, F.G., Cohen, L.B., and Edinton, J.D.: Bowel function of long-term tube-fed patients consuming formulae with and without dietary fiber. J. Parenteral Enteral Nutr., 14, 508-512, 1990.

[45] Patrick, P.G., Gohman, S.M., Marx, S.C., Dclegge, M.H., and Greenberg, N.A.: Effect of supplements of partially hydrolyzed guar gum on the occurrence of constipation and use of laxative agents. J. Am. Diet. Assoc., 98, 912-914, 1998.

[46] Conrad, M.E.: Iron absorption, in Physiology of the Gastrointestinal Tract, $2^{\text {nd }}$ Edition, ed. By Johnson, L.R., Raven Press, New York, pp. 1437-1454, 1987.

[47] Loria, A., Medal, L.S., and Elizondo, J.: Effect of sorbitol on iron absorption in man. Am. J. clin. Nutr., 10, 124-127, 1962.

[48] Jacob, A. and Miles, P.M.: The formation of iron complexes with bile and bile constituents. Gut, 11, 732-734, 1970.

[49] Kelsay, J.L., Behall, K.M., and Praher, E.S.: Effect of fiber from fruits and vegetables on metabolic responses of human subjects. II. Calcium, magnesium, iron and silicon balances. Am. J. Clin. Nutr., 32, 1876-1880, 1979.

[50] Harmuth-Hoene, A.E. and Schelenz, R.: Effect of dietary fiber on mineral absorption in growing rats. J. Nutr., 110, 1774-1784, 1980.

[51] Ikegami, S., Tsuchihashi, F., Harada, H., Tsuchihashi, N., Nishide, E., and Innami, S.: Effect of viscous indigestible polysaccharides on pancreatic-biliary secretion and digestive organs in rats. J. Nutr., 120, 353-360, 1990.

[52] Takahashi, H., Yang, S.I., Ueda, Y., Kim, M., and Yamamoto, T.: Influence of intact and partially hydrolyzed guar gum on iron utilization in rats fed on iron-deficient diets. Comp. Biochem. Physiol., 109A, 75-82, 1994.

[53] Takahashi, H., Akachi, S., Ueda, Y., Kim, M., Hirono, K., and Yamada, T.: Effect of liquid diets with or without partially hydrolyzed guar gum on intestinal microbial flora and function of rats. Nutr. Res., 15, 527-536, 1995.

[54] Hara, H., Nagata, M., Ohta, A., and Kasai, T.: Increases in calcium absorption with ingestion of soluble dietary fibre, guar-gum hydrolysate, depend on the caecum in partially nephrectomized and normal rats. Br. J. Nutr., 76, 773-784, 1996.

[55] Shah, N., Atallah, M.T., Mahoney, R.R., and Pellet, P.L.: Effect of dietary fiber components on fecal nitrogen excretion and protein utilization in growing rats. J. Nutr., 112, 658-666, 1982.

[56] Poksay, K.S. and Schneeman, B.O.: Pancreatic and intestinal response to dietary guar gum in rats. J. Nutr., 113, 15441549, 1983.

[57] Shah, N., Mahoney, R.R., and Pellett, P.L.: Effect of guar gum, lignin and pectin on proteolytic enzyme levels in the gastrointestinal tract of the rat: a time-based study. J. Nutr., 116, 786-794, 1986.

[58] Takahashi, H., Yang, S.I., Kim, M., and Yamamoto, T.: Protein and energy utilization of growing rats fed on the diets containing intact or partially hydrolyzed guar gum. Comp. Biochem. Physiol., 107A, 255-260, 1994.

[59] Ullrich, I.H.: Evaluation of a high-fiber diet in hyperlipidemia: a review. J. Am. Coll. Nutr., 6, 19-25, 1987.
[60] Chen, W.J. and Anderson, J.W.: Effects of guar gum and wheat bran on lipid metabolism of rats. J. Nutr., 109, 10281034, 1979.

[61] Jenkins, D.J.A., Wolever, T.M.S., Leeds, A.R., Gassull, M.A., Haisman, P., Dilawari, J., Goff, D.V., Metz,G.L., and Alberti, K.G.M.M.: Dietary fibers, fiber analogues and glucose tolerance: importance of viscosity. Br. Med. J., 1, 1392-1394, 1978.

[62] Salvioli, G., Mambrini, A., and Lugli, R.: Effects of dietary fibers on cholesterol absorption and pancreatic enzyme (lipase), in New Trends in Pathophysiology and Therapy of Large Bowel, eds. By Barbara, L., Miglioli, M., and Phillips, S.F., Elsevier Science Publishers B.V., pp. 275-282, 1983.

[63] Anderson, J.W. and Bryant, C.A.: Dietary fiber: Diabetes and obesity. Am. J. Gastroenterol., 81, 898-906, 1986.

[64] Aro, A., Uusitupa, M., Voutilainen, E., Hersio, K., Korhonen, T., and Sittonen, O.: Improved diabetic control and hypocholesterolemic effect induced by long-term dietary supplementation with guar gum in type II (Insulin independent) diabetes. Diabetologia, 21, 29-33, 1981.

[65] Kiriyama, S., Okazaki, Y., and Yoshida, A.: Changes in hypocholesterolemic activity in rats by various konjak powder treatments. Agric. Biol. Chem., 34, 641-643, 1970.

[66] Yamashita, K., Kowai, K., and Itakura, K.: Effect of fructooligosaccharides on blood glucose and serum lipids in diabetic subjects. Nutr. Res., 4, 961-966, 1984.

[67] Yamatoya, K., Kuwano, K., and Suzuki, J.: Effects of hydrolyzed guar gum on cholesterol and glucose in humans. Food Hydrocolloids, 11, 239-242, 1997.

[68] Hunt, S.M. and Groff, J.L.: Advanced nutrition and human metabolism. West Publishing Company, St. Paul, MN, U.S.A, 1990.

[69] Velazquez, M., Davies, C., Marett, R., Slavin, J.L., and Feirtag, J.M.: Effect of oligossaccharides and fibre substitutes on short-chain fatty acid production by human faecal microflora. Anaerobe, 6, 87-92, 2000.

[70] Scott, R.W., Anderson, J.W., and Bridges, S.R.: Propionate inhibits hepatocyte lipid synthesis. Proc. Soc. Exp. Biol. Med., 195, 26-29, 1990.

[71] Cummings, J.H. and Englyst, H.N.: Fermentation in the human large intestine and available substrates. Am. J. Clin. Nurt., 45, 1243-1255, 1987.

[72] Ek, A.C., Larsson, J., and von Schenck, H.: The correlation between energy, malnutrition and clinical outcome in an elderly hospital population. Clin Nutr., 9, 185-189, 1990.

[73] Jenkins, D.J.A., Leeds, A.R., and Gassull, M.A.: Decrease in post-prandial insulin and glucose concentrations for guar and pectin. Ann. Intern. Med., 86, 20-23, 1977.

[74] Golay, A., Schneider, H., Bloese, D., Vadas, L., and Assal, J.: The effect of a liquid supplement containing guar gum and fructose on glucose tolerance in non-insulin-dependent diabetic patients. Nutr. Metab. Cardiovasc. Dis., 5, 141-148, 1995.

[75] Wolever, T., Jenkins, D.J.A., Nineham, R., and Alberti, D.G.: Guar gum and reduction of postprandial glycaemia: Effect of incorporation into solid food, liquid food and both. Br. J. Nutr., 41, 505-510, 1979. 
[76] Yamatoya, K., Dekiya, K., Yamada, H., and Ichikawa, T.: Effects of partially hydrolyzed guar gum on postprandial plasma glucose and lipid levels in humans. J. Jpn. Soc. Nutr. Food Sci., 46, 199-203, 1993.

[77] Tsuda, K., Inden, T., Yamanaka, K., and Ikeda, Y.: Effect of partially hydrolyzed guar gum on elevation of blood glucose after sugar intake in human volunteers. Jpn. J. Diet. Fib., 2, 15-22, 1998.

[78] Morgan, L,M., Goulder, T.J., and Tsiolakis, D.: The effect of unabsorbable carbohydrate on gut hormones. Diabetologia, 17, 85-89, 1979.

[79] Wilson, I.G., Heaney, J.C., and Powel, G.G.: Salmonella in raw shell egg in Northern Ireland: 1996-1997. Commun. Diseases Public Health, 1, 156-160, 1998.

[80] Konuma, H., Shinagawa, K., and Kumagai, S.: Salmonella food poisoning and hen eggs. Modern Media, 38, 59-64, 1995.
[81] Hennessy, T.W., Hedberg, C.W., Slutsker, L., White, K.E., Besser-Wiek, J.M., Moen, M.E., Feldman, J., and Coleman, M.T.: A national outbreak of Salmonella enteritides infections from ice cream. New Eng. J. Med., 334, 1281-1286, 1996.

[82] Dodhia, H., Hearney, J., and Warburton, F.: A birthday party, home-made ice cream, and an outbreak of salmonella enteritidis phage type 6 infection. Commun. Diseases Public Health, 1, 31-34, 1998.

[83] Oyofo, B.A., Deloach, J.R., Corrier, D.E., Norman, J.O., Ziprin, R.L., and Molleenhauer, H.H.: Prevention of Salmonella typhimurium colonization of broilers with D-mannose. Poultry Science, 68, 1357-1360, 1989.

[84] Line, J.E., Bailey, J.S., Cox, N.A., Stern, N.J., and Tompkins, T.: Effect of yeast-supplemented feed on Salmonella and Campylobacter populations in broilers. Poultry Science, 77, 405-410, 1998. 